\title{
B cells as biomarkers: predicting immune checkpoint therapy adverse events
}

\author{
Shannon M. Liudahl and Lisa M. Coussens \\ Department of Cell, Developmental and Cancer Biology and the Knight Cancer Institute, Oregon Health and Science University (OHSU), Portland, Oregon, USA.
}

\begin{abstract}
Immune checkpoint inhibitors are becoming a cornerstone of cancer immunotherapy as a result of their clinical success in relieving immune suppression and driving durable antitumor $\mathrm{T}$ cell responses in certain subsets of patients. Unfortunately, checkpoint inhibition is also associated with treatment-related toxicities that result in a myriad of side effects, ranging from mild and manageable to severe and debilitating. In this issue of the $J C l$, Das and colleagues report an association between early therapy-induced changes in circulating $B$ cells and an increased risk of high-grade immune-related adverse events (IRAEs) in patients treated with checkpoint inhibitors that target cytotoxic T lymphocyte-associated antigen-4 (CTLA4) and programmed cell death protein 1 (PD1). These findings identify potential predictive biomarkers for high-grade IRAEs that may be leveraged to improve patient monitoring and may prompt new treatment strategies to prevent IRAEs.
\end{abstract}

Checkpoint blockade therapy: the good, the bad, and the toxic FDA approval of the use of immune checkpoint inhibitors for melanoma, head and neck cancer, non-small-cell lung cancer, urothelial carcinoma, and renal cell carcinoma has transformed clinical oncology within the past decade, and this class of therapies continues to undergo extensive evaluation for the treatment of a broad spectrum of additional tumor types. The principal goal of checkpoint inhibition is to bolster $\mathrm{CD}^{+} \mathrm{T}$ cell cytotoxic effector function by relieving inhibitory brakes that, while critical for maintaining selftolerance, prevent optimal $\mathrm{T}$ cell activation in response to malignancy (1). The substantial promise of checkpoint inhibition is reflected in the improved survival outcomes observed in the CheckMate 067 clinical trial (ClinicalTrials.gov NCT01844505) that evaluated combination treatment with ipilimumab, an anti-cytotoxic T lymphocyteassociated antigen-4-targeted (CTLA4targeted) $\mathrm{mAb}$, and nivolumab, a mAb targeting programmed cell death protein 1 (PD1), in patients with previously untreated advanced melanoma (2). Patients who received combination therapy or nivolumab monotherapy had three-year overall survival rates of $58 \%$ and $52 \%$, respectively, with $19 \%$ of patients in the combination arm showing complete responses (2).

Despite the undeniable clinical success of anti-CTLA4 and anti-PD1 mAb therapy thus far, especially in highly immunogenic cancers such as melanoma, several challenges remain. A current paucity of biomarkers limits the ability to predict who will respond to these immune therapies (3) and, importantly, who will develop treatment-related autoimmune toxicity,

Related Article: p. 715

Conflict of interest: L.M. Coussens is a member of the External Advisory Board for Syndax Pharmaceuticals Inc., has sponsored research agreements with Deciphera Pharmaceuticals Inc., Syndax Pharmaceuticals Inc., Acerta Pharma BV, and Roche Glycart AG, and has filed for patent protection under US Patent Publication numbers 2008-0206219, 2010 276324, and 2017-0160171.

Reference information: / Clin Invest. 2018;128(2):577-579. https://doi.org/10.1172/JCI99036.

which is a serious concern for the majority of treated patients. Such toxicities, known as immune-related adverse events (IRAEs), vary in severity and in the organ systems affected. Patients receiving checkpoint inhibitors have a significantly higher risk of developing IRAEs than do patients receiving other forms of therapy, and combined anti-CTLA4 and anti-PD1 mAb therapy leads to a higher incidence of all-grade and high-grade (grade $\geq 3$ ) IRAEs than does either agent alone $(2,4-6)$.

IRAEs associated with anti-CTLA4 and anti-PD1 mAb therapy most commonly impact the skin, gastrointestinal, and endocrine systems and manifest as a variety of conditions, such as rash, pruritus, vitiligo, diarrhea, colitis, and thyroid dysregulation (7). Although IRAE symptoms are usually manageable and reversible, they frequently result in either treatment interruption or dose reduction and/or discontinuation of checkpoint therapy. As an example of the prevalence and challenge of IRAEs, $96 \%$ of patients in the ipilimumab and nivolumab combination arm of the CheckMate 067 trial experienced at least one IRAE (any grade), with $30 \%$ of these patients discontinuing treatment as a direct consequence of their IRAEs (2). Although it has been reported that neither IRAE management with immune-suppressive corticosteroids nor discontinuation of therapy because of IRAEs markedly interferes with a durable clinical response to anti-CTLA4 and anti-PD1 mAbs $(2,8)$, strategies to reduce patient morbidity stemming from IRAEs are desirable. Unfortunately, the specific immune mechanism(s) that drive IRAEs are unclear, and clinical strategies to predict and prevent high-grade IRAEs are lacking.

\section{Circulating B cell abundance correlates with IRAE risk}

$\mathrm{T}$ and $\mathrm{B}$ lymphocytes are critical mediators of autoimmunity and are thus implicated in IRAE pathogenesis. Recent studies have revealed that changes in circulating $\mathrm{T}$ cell repertoires in ipilimumab-treated patients 


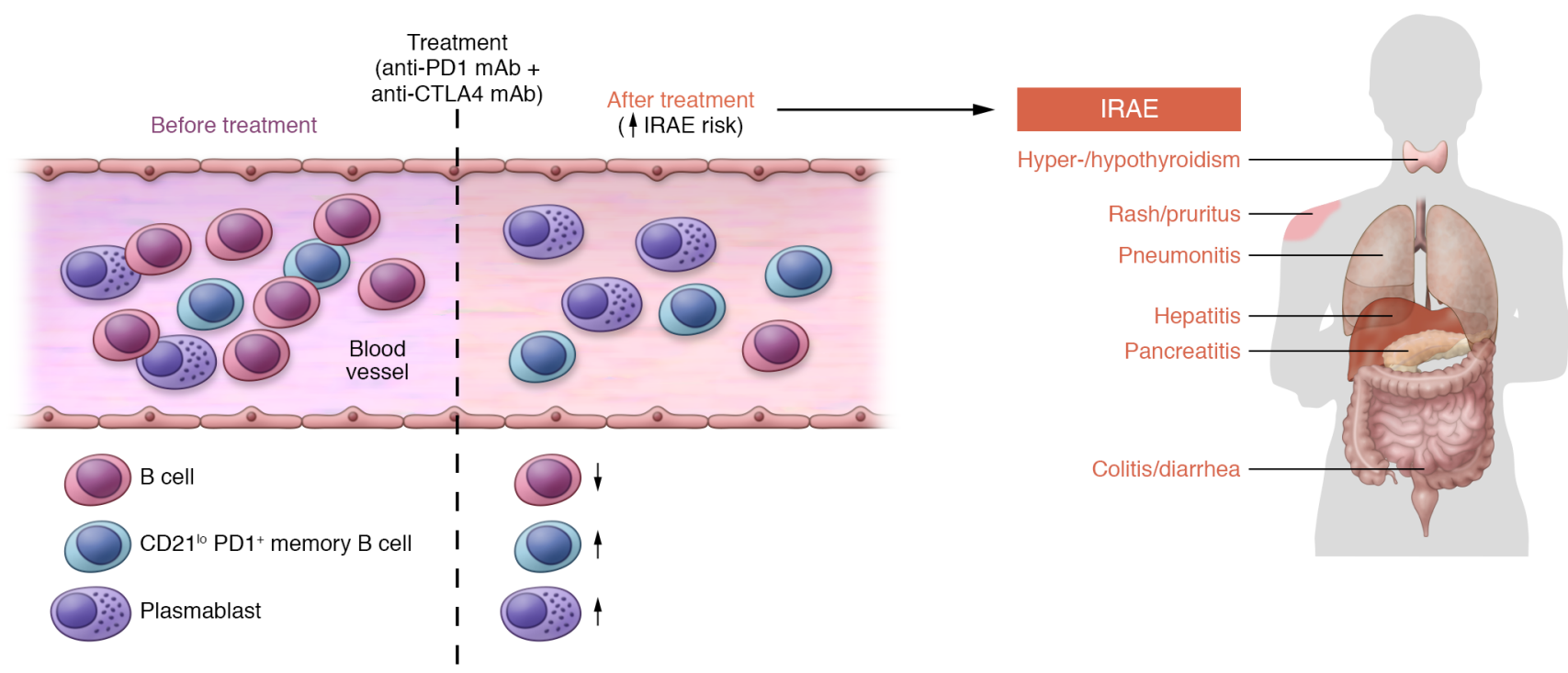

Figure 1. Changes in circulating B cells predict IRAE risk in patients receiving combined anti-CTLA4 and anti-PD1 therapy. Patients showing changes in circulating B cells after one cycle of combination anti-CTLA4 and anti-PD1 therapy (compared with their pretreatment baseline) have an increased risk of developing high-grade IRAEs. Specifically, a post-treatment reduction in total peripheral B cells and a coincident enrichment of differentiated CD21 ${ }^{10} \mathrm{PD} 1^{+}$memory B cells and plasmablasts correlate with subsequent IRAE development. B cell changes are a unique immune biomarker of IRAE risk, as early changes in the frequency of other circulating leukocyte populations were not detected after therapy (data not shown). Select high-grade IRAE pathologies associated with combined anti-CTLA4 and anti-PD1 mAb therapy are depicted, and patients who showed B cell changes had a median three-week time to onset of one or more such IRAEs.

preceded the development of IRAEs (9, 10). While changes in $\mathrm{T}$ cell genomic signatures in patients undergoing antiCTLA4 and anti-PD1 mAb treatment have also been identified (11), changes in B cells during checkpoint inhibition have not been previously reported.

In this issue, Das et al. analyzed circulating B cells in a small cohort of patients with advanced melanoma before and after treatment with anti-CTLA4 and anti-PD1 mAbs, administered as single agents or in combination (12). They found that a reduction in total peripheral $\mathrm{B}$ cells after a single cycle of combined checkpoint blockade (CCB) coincided with enrichment of plasmablasts and a proliferative $\mathrm{CD} 21^{\text {lo }} \mathrm{PD} 1^{+}$memory $\mathrm{B}$ cell subset. Single-cell RNA sequencing of CD2 $1^{\text {lo }} \mathrm{PD} 1^{+}$ $\mathrm{B}$ cells collected from a patient prior to and after $\mathrm{CCB}$ revealed increased transcription of genes associated with cell activation and inflammatory cytokine production following treatment. $\mathrm{CD} 21^{\text {10 }}$ B cells also expressed lower levels of the lymphoid tissue-homing chemokine receptors CXCR4 and CXCR5 as compared with $\mathrm{CD} 21^{\mathrm{hi}} \mathrm{B}$ cells, indicating that $\mathrm{CD} 21^{\text {lo }}$ cells may have a greater capacity to traffic to nonlymphoid tissues and contribute to inflammatory processes that may mediate autoimmunity.
Given these findings, Das et al. developed a metric to evaluate whether changes in the frequency of circulating $B$ cells in CCB-treated patients correlated with an increased risk or severity of IRAEs. Using this metric, the authors found that patients with a $30 \%$ or greater reduction in baseline levels of total circulating B cells and a twofold or greater increase in $\mathrm{CD} 21^{\text {1o }} \mathrm{B}$ cells or plasmablasts were significantly more likely to develop high-grade IRAEs than were patients without B cell changes (Figure 1) Moreover, early changes in circulating B cells after only one round of $\mathrm{CCB}$ correlated with a median time of three weeks to IRAE onset. Importantly, changes in the frequency of other circulating immune cell populations, including $\mathrm{T}$ cells, before and after therapy did not correlate with the development of IRAEs.

\section{Clinical implications and future directions}

Together, findings from Das and colleagues indicate that changes in circulating $B$ cells may be useful predictors of IRAE risk (12). Clinical application of B cell monitoring could lead to earlier IRAE intervention and reduced IRAE severity, both of which would ideally translate to a reduced discontinuation of checkpoint therapy. The sample size in this study was limited, thus, a critical next step will be to determine the robustness of the proposed B cell signature in expanded patient cohorts. Significant changes in both total B cell frequency and the frequency of $\mathrm{CD} 21^{\text {1o }} \mathrm{B}$ cells or plasmablasts were only observed in the CCB group, indicating that patients undergoing combination therapy may preferentially benefit from B cell monitoring. However, future evaluation of larger cohorts will reveal whether subsets of patients receiving monotherapy undergo similar B cell changes equally predictive of IRAE risk. It will also be necessary to determine whether changes in circulating B cells occur specifically in melanoma, or whether this signature is also detectable in patients with other tumor types.

The mechanistic contribution of $\mathrm{B}$ cells to IRAEs also remains unclear. While the B cell changes observed in CCB-treated patients did not correlate with the clinical response to therapy (12), it remains to be determined whether and how B cells directly mediate IRAEs. B cell receptor (BCR) sequencing of total B cells revealed post-therapy clonal expansion in a subset of patients in the CCB and monotherapy groups, but this did not correlate with the expansion of a single dominant clone, thus arguing against $\mathrm{B}$ cell-mediated autoreactivity against a discrete self-antigen (12). Additional studies will be required to determine the functional relevance of 
$\mathrm{CD} 21^{\text {lo }}$ memory B cells and plasmablasts in IRAE pathogenesis.

Despite the outstanding questions regarding specific $B$ cell mechanisms in IRAEs, B cell monitoring represents a relatively simple, noninvasive clinical biomarker assessment strategy that could also yield preventative benefits. Circulating biomarkers for treatment-related toxicities in other forms of immunotherapy have recently been identified and are poised to have clinical impact. For example, in cancer patients receiving chimeric antigen receptor $\mathrm{T}$ cell (CAR-T cell) therapy, early elevation of specific serum cytokines and other soluble factors, including IFN- $\gamma$, MIP1 $\alpha$, IL-6, and soluble gp130 (sgp130), precedes the development of severe cytokine release syndrome (CRS) $(13,14)$. Use of the IL-6 receptor (IL-6R) inhibitor tocilizumab is now approved for the treatment of severe CRS in CAR-T cell recipients, and the identification of circulating biomarkers that predict CRS may lead to prophylactic administration of tocilizumab or other cytokine inhibitors in patients who have markers associated with increased risk. Similarly, B cell changes as a biomarker for IRAEs in checkpoint inhibition therapy could lead to new preventative strategies. Along these lines, Das and colleagues suggest the potential utility of B cell-targeted therapies as a preventative measure against IRAEs. This idea is compelling, especially given the clinical success of B cell-depleting antibodies and inhibitors of Bruton's tyrosine kinase (BTK), an essential kinase for B cell maturation and signaling, for treating autoimmune diseases (15) and graft-versus-host disease (16).

It is likely that, in addition to reducing the risk of IRAEs, B cell depletion or BTK inhibition may also enhance the antitumor efficacy of checkpoint inhibitors, at least in some settings. The role of B cells in melanoma progression is controversial, as both proand antitumor B cell functions have been reported (17); however, the results from the use of B cell depletion in a small cohort of melanoma patients appear promising (18). Recent studies of other solid tumors have identified various B cell subsets as critical protumoral mediators of malignancy (1925), and the enriched B cell populations identified by Das and colleagues may share similar functional properties. If so, these findings would support B cell depletion or BTK inhibition along with checkpoint inhibition as an appealing strategy to further explore. In fact, a clinical trial involving patients with head and neck squamous cell carcinoma is currently evaluating this approach (ClinicalTrials.gov NCT02454179). Time will tell whether such combinations simultaneously enhance antitumor immunity, limit IRAEs, and improve clinical outcomes. What is clear for now is that, although $\mathrm{T}$ cell responses are often the main focus of immunotherapy, B cells should not be overlooked.

\section{Acknowledgments}

The authors acknowledge support from a Department of Defense (DOD) Breast Cancer Research Program (BCRP) Era of Hope Scholar Expansion Award (W81XWH-08PRMRP-IIRA); the Susan G. Komen Foundation (KG110560); the Breast Cancer Research Foundation; a Stand Up to Cancer - Lustgarten Foundation Pancreatic Cancer Convergence Dream Team Translational Research Award; the OHSU Brenden-Colson Center for Pancreatic Care; and the OHSU Knight Cancer Institute.

Address correspondence to: Lisa M. Coussens, Department of Cell, Developmental \& Cancer Biology, Knight Cancer Institute, Oregon Health \& Science University, 3181 SW Sam Jackson Park Road, Portland, Oregon 97239-3098, USA. Phone:503.494.7811; Email: coussenl@ohsu.edu.

1. Topalian SL, Drake CG, Pardoll DM. Immune checkpoint blockade: a common denominator approach to cancer therapy. Cancer Cell. 2015;27(4):450-461.

2. Wolchok JD, et al. Overall survival with combined nivolumab and ipilimumab in advanced melanoma. NEngl JMed. 2017;377(14):1345-1356.

3. Nishino M, Ramaiya NH, Hatabu H, Hodi FS. Monitoring immune-checkpoint blockade: response evaluation and biomarker development. Nat Rev Clin Oncol. 2017;14(11):655-668.

4. El Osta B, Hu F, Sadek R, Chintalapally R, Tang SC. Not all immune-checkpoint inhibitors are created equal: meta-analysis and systematic review of immune-related adverse events in cancer trials. Crit Rev Oncol Hematol. 2017;119:1-12.

5. De Velasco G, et al. Comprehensive Meta-analysis of key immune-related adverse events from CTLA-4 and PD-1/PD-L1 inhibitors in cancer patients. Cancer Immunol Res. 2017;5(4):312-318.

6. Larkin J, et al. Combined nivolumab and ipilimumab or monotherapy in untreated melanoma. N Engl J Med. 2015;373(1):23-34.

7. Stucci S, et al. Immune-related adverse events during anticancer immunotherapy: pathogenesis and management. Oncol Lett. 2017;14(5):5671-5680.
8. Horvat TZ, et al. Immune-related adverse events, need for systemic immunosuppression, and effects on survival and time to treatment failure in patients with melanoma treated with ipilimumab at Memorial Sloan Kettering Cancer Center. J Clin Oncol. 2015;33(28):3193-3198.

9. Subudhi SK, et al. Clonal expansion of CD8 T cells in the systemic circulation precedes development of ipilimumab-induced toxicities. Proc Natl Acad Sci U S A . 2016;113(42):11919-11924.

10. Oh DY, et al. Immune toxicities elicted by CTLA4 blockade in cancer patients are associated with early diversification of the T-cell repertoire. Cancer Res. 2017;77(6):1322-1330.

11. Das R, et al. Combination therapy with antiCTLA-4 and anti-PD-1 leads to distinct immunologic changes in vivo. J Immunol. 2015;194(3):950-959.

12. Das R, et al. Early B cell changes predict autoimmunity following combination immune checkpoint blockade. J Clin Invest. 2018;128(2):715-720.

13. Teachey DT, et al. Identification of predictive biomarkers for cytokine release syndrome after chimeric antigen receptor T-cell therapy for acute lymphoblastic leukemia. Cancer Discov. 2016;6(6):664-679.

14. Hay KA, et al. Kinetics and biomarkers of severe cytokine release syndrome after CD19 chimeric antigen receptor-modified T-cell therapy. Blood. 2017;130(21):2295-2306.

15. Gürcan HM, Keskin DB, Stern JN, Nitzberg MA, Shekhani H, Ahmed AR. A review of the current use of rituximab in autoimmune diseases. Int Immunopharmacol. 2009;9(1):10-25.

16. Miklos D, et al. Ibrutinib for chronic graft-versushost disease after failure of prior therapy. Blood. 2017;130(21):2243-2250.

17. Chiaruttini $\mathrm{G}$, et al. B cells and the humoral response in melanoma: The overlooked players of the tumor microenvironment. Oncoimmunology. 2017;6(4):e1294296.

18. Somasundaram R, et al. Tumor-associated $\mathrm{B}$-cells induce tumor heterogeneity and therapy resistance. Nat Commun. 2017;8(1):607.

19. Affara NI, et al. B cells regulate macrophage phenotype and response to chemotherapy in squamous carcinomas. Cancer Cell. 2014;25(6):809-821.

20. Shalapour $S$, et al. Immunosuppressive plasma cells impede T-cell-dependent immunogenic chemotherapy. Nature. 2015;521(7550):94-98.

21. Gunderson AJ, et al. Bruton tyrosine kinase-dependent immune cell cross-talk drives pancreas cancer. Cancer Discov. 2016;6(3):270-285.

22. Pylayeva-Gupta Y, et al. IL35-producing B cells promote the development of pancreatic neoplasia. Cancer Discov. 2016;6(3):247-255.

23. Lee KE, et al. Hifla deletion reveals pro-neoplastic function of B cells in pancreatic neoplasia. Cancer Discov. 2016;6(3):256-269.

24. Xiao X, et al. PD-1 ${ }^{\text {hi }}$ identifies a novel regulatory $\mathrm{B}$-cell population in human hepatoma that promotes disease progression. Cancer Discov. 2016;6(5):546-559.

25. Shalapour $\mathrm{S}$, et al. Inflammation-induced $\operatorname{Ig} \mathrm{A}^{+}$ cells dismantle anti-liver cancer immunity. Nature. 2017;551(7680):340-345 